\title{
Primary Limited Lumbar Discectomy with an Annulus Closure Device: One-Year Clinical and Radiographic Results from a Prospective, Multi-Center Study
}

\author{
Michiel B. Lequin', Martin Barth ${ }^{2}$, Claudius Thomé ${ }^{3}$, Gerrit J. Bouma \\ ${ }^{I}$ Department of Neurosurgery, Sint Lucas-Andreas Ziekenhuis and Academic Medical Center, Amsterdam. The Netherlands \\ ${ }^{2}$ Department of Neurosurgery, University Hospital Mannheim, Mannheim, Germany \\ ${ }^{3}$ Department of Neurosurgery, Medical University Innsbruck, Innsbruck, Austria
}

Objective: Discectomy as a treatment for herniated lumbar discs results in outcomes after surgery that are not uniformly positive. Surgeons face the dilemma between limited nucleus removal which is associated with a higher risk of recurrence, or more aggressive nucleus removal which may lead to disc height loss and persistent back-pain. annulus closure devices may allow for the benefits of limited nucleus removal without the increased risk of recurrence. This is an interim report of an ongoing 24month post-marketing study of the Barricaid ${ }^{\circledR}$ annulus closure device, consisting of a flexible polymer mesh that blocks the defect, held in place by a titanium bone anchor.

Methods: We prospectively enrolled 45 patients at four hospitals, and implanted the Barricaid ${ }^{\circledR}$ after a limited discectomy. annulus defect size and volume of removed nucleus were recorded. Reherniations were reported, pain and function were monitored and imaging was performed at regular intervals during 24 months of follow-up.

Results: At 12 months postsurgery, pain and function were significantly improved, comparing favorably to reported results from limited discectomy. Disc height has been well maintained. One reherniation has occurred $(2.4 \%)$, which was associated with a misplaced device. No device fracture, subsidence or migration has been observed.

Conclusion: The use of an annulus closure device may provide a reduction in reherniation rate for lumbar discectomy patients with large annulus defects who are at the greatest risk of recurrence. Using such a device should provide the surgeon increa- sed confidence in minimizing nucleus removal, which, in turn, may preserve disc height and biomechanics, reducing dege- neration and associated poor clinical outcomes in the long-term. A randomized multicenter study evaluating limited discectomy with and without the Barricaid ${ }^{\circledR}$ is currently underway, and will provide a higher level of evidence.

Key Words: Discectomy • Disc herniation • Annulus fibrosus • Annulus closure device

\section{INTRODUCTION}

Lumbar discectomy is the most common surgical procedure performed for patients experiencing back and leg pain. Despite high rates of safety and success in relieving pain and improving function, between $10 \%$ and $30 \%$ of discectomy patients

\footnotetext{
- Received: December 4, 2012 • Revised: December 16, 2012

- Accepted: December 24, 2012

Corresponding Author: Gerrit J. Bouma, MD, PhD

Department of Neurosurgery, Academic Medical Center,

P.O. Box 226601100 DD Amsterdam

Tel: 31-20-5663542, Fax: 31-20-4001849

Email: g.j.bouma@amc.uva.nl

${ }^{*}$ Clinical Trial Registration: clinicaltrials.gov ID NCT01534065

*Disclosure: Patient follow-up and data analysis was financially supported by Intrinsic Therapeutics, Inc. None of the authors have a financial interest in any of the products or companies discussed.
}

continue to experience unsatisfactory results ${ }^{6,7,8,21)}$. Loss of disc height ${ }^{4,27)}$ and recurrent disc herniation ${ }^{7,16}$ have been identified as important factors contributing to poor outcomes following lumbar discectomy.

To prevent reherniation, traditionally a subtotal discectomy has been recommended with removal of as much of the disc nucleus as possible. This practice, however, leads to increased disc disruption with loss of disc height, which will transfer axial loads radially to the annulus fibrosus and to the facet joints of the posterior column, thus contributing to long-term back pain $^{1,10)}$.

Attempting to minimize these effects, a limited discectomy procedure has been proposed, aiming to remove only the offending disc fragment and any loose disc material without further invading the disc space. Limited discectomy, however, is associated with a greater incidence of recurrent disc hernia- 
tion $^{7,17,26)}$.

Carragee et al. showed that the rate of reherniation after limited discectomy was greatest $(27.3 \%)$ in patients with annulus defects wider than $6 \mathrm{~mm}$, and suggested that a barrier to close the defect in these patients may be clinically useful in preventing reherniation ${ }^{5}$. In addition, by reinforcing the disc annulus, such a barrier may also contribute to maintenance of disc pressure and height, thus preserving disc function and possibly retarding further degeneration of the disc and facet joints.

Previous attempts to close the annulus defect have failed because they relied on the strength and stability of the remaining annulus which usually falls short ${ }^{13)}$. Recently, an annulus closure device was developed consisting of a flexible polyester mesh to close the defect. The mesh is attached to a titanium bone anchor implanted into the adjacent vertebral body, providing stable footing for the implant that is better able to withstand the high pressures within the disc.

We prospectively evaluated the safety and efficacy of this new annulus closure device when used as an adjunct to primary limited discectomy in patients with symptomatic disc herniation refractory to conservative treatment. In this paper, the first 12 months of follow-up of this 24-month study are reported.

\section{MATERIALS AND METHODS}

This multi-center, prospective study (clinicaltrials.gov ID NCT01534065) was initiated with Medical Ethics Committee approval for each site. Forty-five patients were enrolled in this study. Patients who in the surgeon's opinion seemed eligible for this study were asked to participate and screened for eligibility. All patients had sciatica due to an MRI-confirmed lumbar disc herniation unresponsive to conservative therapy for at least 6 weeks. Further select inclusion and exclusion criteria for study participations are listed in Table 1 .

Posterior lumbar discectomy with limited nucleus removal and implantation of the annulus closure device was performed in the patients at four sites. After informed consent, all subjects were assessed preoperatively and postoperatively at 6 weeks and at 3, 6, and 12 months. Low back and radicular leg pain were evaluated using a visual analog scale (VAS). The Oswestry Disability Index (ODI) was administered to assess function. Antero-posterior, neutral lateral, and flexion-extension radiographs were obtained at all evaluation intervals. Multiplanar computed tomography (CT) and magnetic resonance imaging (MRI) were obtained preoperatively and postoperatively at 12 months. Analysis by an independent radiology lab (Medical Metrics, Inc.; Houston, TX, USA) was conducted to assess radiographic data, including all MR images to identify asymptomatic recurrent herniations. Preoperative and postoperative disc space height at the involved level was measured at the anterior and posterior edges of the endplates using neutral lateral radiographs ${ }^{23}$. Postoperative radiographs also were examined for evidence of heterotopic bone formation (bridging of trabecular bone/fusion between the vertebrae), device subsidence/migration, and device condition (intact or fractured). In addition, the degree of facet arthropathy and disc degeneration was assessed using validated grading scales ${ }^{20,22}$.

Table 1. Selected inclusion and exclusion criteria for study participation.

Select Inclusion Criteria

1. Age 18 to 75 years old.

2. Posterior or posterolateral disc herniations at one or two levels between L1 and S1 with radiographic confirmation of neural compression using CT and/or MRI.

3. At least six weeks of failed, conservative treatment prior to surgery, including physical therapy, use of anti-inflammatory medications at maximum specified dosage, and/or administration of epidural/facet injections.

4. Minimum posterior disc height of $3 \mathrm{~mm}$ at the index level(s).

5. Oswestry Disability Index score of at least $40 / 100$ at baseline.

6. VAS leg pain of at least $40 / 100$ at baseline.

\section{Select Exclusion Criteria}

1. Spondy lolisthesis Grade II or higher.

2. Requires uni- or bilateral facetectomy to treat leg/back pain.

3. Back or non-radicular leg pain of unknown etiology.

4. Prior disc surgery at the index lumbar vertebral level, including fusion (with or without instrumentation), motion preservation, facetectomy, or intradiscale lectrothermal therapy.

5. Requires a spine DEXA (i.e., subjects with SCORE of $\geq 6$ ) with a T-score less than -2.0 at the index level.

6. Clinically compromised vertebral bodies at the index level(s) due to any traumatic, neoplastic, metabolic, or infectious pathology.

7. Morbid obesity (defined as a body mass index $>40$, or weighs more than $100 \mathrm{lbs}$ over ideal body weight). 
Complications and subsequent surgeries were monitored and documented throughout the study. The study was monitored by an independent Data Safety Monitoring Board who reviewed all safety-related issues, including adverse events and protocol violations (see appendix).

Preoperative clinical data were compared to postoperative data at 6 weeks, 3 months, 6 months, and 12-months. For contin uous, paired variables that were normally distributed, a paired t-test was used to determine statistical significance. Wilcoxon Signed Rank Test was used to compare paired, ordinal variables or continuous, paired variables that were not normally distributed. Missing data were not estimated or carried forward in any statistical analyses. Statistical differences were considered significant when the p-value was less than or equal to 0.05 . Statistical analysis was performed using SigmaStat ${ }^{\circledR}$ Software, version 2.0 (SPSS, Inc., Chicago, Illinois).

\section{Device Description}

The Barricaid ${ }^{\circledR}$ (Intrinsic Therapeutics, Inc., Woburn, MA, USA) annulus closure device was designed as an adjunct to lumbar discectomy to block the annulus defect and maintain nucleus within the disc space. The device has received CEmarking and is comprised of two components: a flexible mesh that prevents migration of the nucleus from within the disc, and a bone anchor which secures the occlusion component to one of the adjacent vertebral bodies (Figs. 1 and 2). The anchor is composed of Ti6Al-4V ELI, a typical orthopedic alloy with a long history of use in permanent implants. The mesh is made up of woven polyester ("Dacron"), which has been in use for implantation as cardiovascular graft material in humans for several decades.

At the time of the study, the implant was available in one size capable of blocking an annulus defect up to $10 \mathrm{~mm}$ wide.

\section{Surgical Technique}

Each surgery was carried out by one of eight neurosurgeons, all well-experienced in lumbar disc surgery. Prior to study

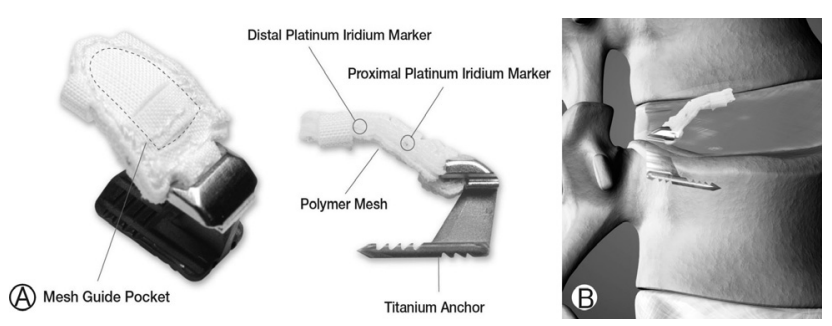

Fig. 1. Barricaid annulus closure device (A) and in situ representation (B). initiation, surgeons took an instructional course which included "hands-on" training of inserting the Barricaid ${ }^{\circledR}$ device in cadaver specimens or in an artificial lumbar spine, though none had implanted any in living humans. In all subjects, a limited discectomy was performed. Any disc material removed, either from within or outside the disc space, was placed dry in a syringe and the volume was measured and recorded. After completion of the discectomy, the size of the annulus defect was measured using sizing probes of $1 \mathrm{~mm}$ increments. If the defect was taller than $6 \mathrm{~mm}$ or wider than $10 \mathrm{~mm}$, implantation of the annulus closure device was not attempted.. In no case, however, the defect exceeded these size limits. Subsequently, a sizing trial, designed to replicate the size and shape of the loaded delivery tool, was used to confirm access through the lamina and the appropriate angle of approach to the disc. Then the delivery tool was placed in the disc under
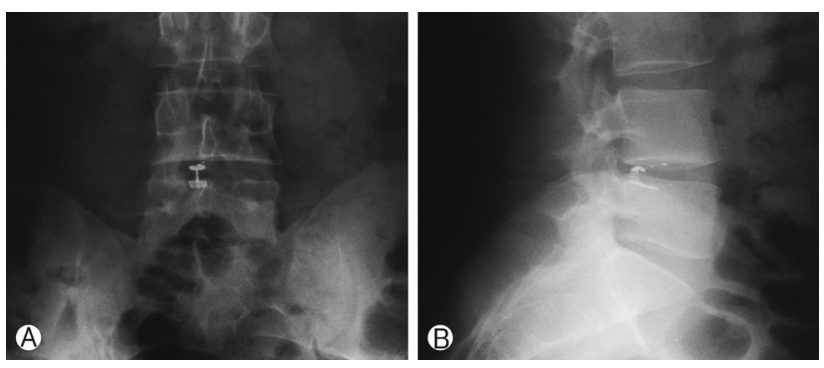

Fig. 2. AP (A) and lateral (B) X-rays of Barricaid at two years.

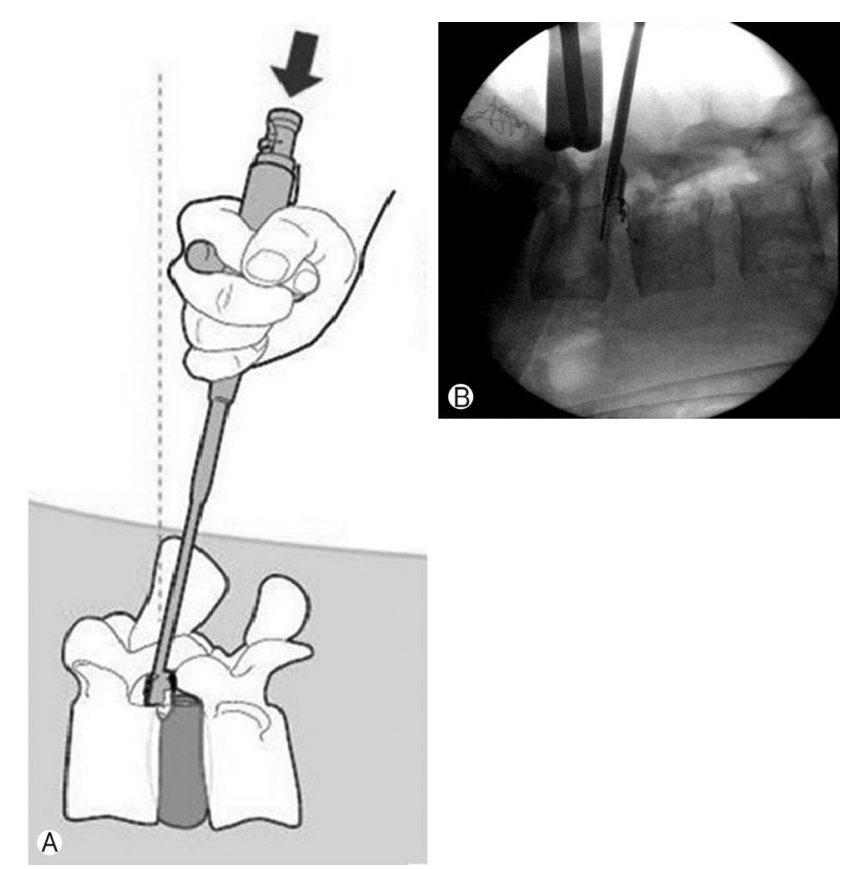

Fig. 3. Diagram (A) and intraoperative fluoroscopy image (B) showing insertion of the Barricaid device. 
fluoroscopic control, ensuring that the angle of approach was parallel to the target endplate in the region of implantation. Holding the delivery tool firmly in position, the anchor was hammered into the endplate, thereby simultaneously placing the mesh in position within the disc (Fig. 3).

Patients were discharged and given post-surgery care instructions per each hospital's standard regimen following lumbar discectomy, without any additional bracing or other activity restrictions.

\section{RESULTS}

Between April 2009 and July 2010, 45 patients provided written informed consent and were enrolled in the study. The baseline, demographic, clinical, radiographic and intra-operative characteristics of the study population are provided in Table 2. One subject had a concomitant musculoskeletal condition (arthritis), and one subject had a previous back procedure, a unilateral decompression at another level.

A flowchart depicting subject accounting is provided in Fig. 4. One subject was not implanted due to a technical error, one subject was excluded from analysis because of an intraoperative protocol violation, and two subjects failed to return for the 12-month follow-up. One patient's implant was removed after approximately 6 months as described in detail below, thus leaving 40 subjects for comparative analysis of pre- and post-operative patient-reported clinical data. One subject was included in the study despite having a VAS-leg $<40(35 / 100)$.

\section{Clinical results}

Table 2. Baseline study population and surgical data (mean \pm SD)

\begin{tabular}{lc}
\hline \hline Demographic variables & $24: 21$ \\
Male: Female ratio & $42.3 \pm 11.4$ \\
Age, years & $26.0 \pm 4.9$ \\
BMl & $49 \%$ \\
Reported smoking & \\
\hline Surgery & $120.2 \pm 31.2$ \\
Length of surgery (min) & $73.8 \pm 66.6$ \\
Estimated blood loss (mL) & \\
Operated level & 2 \\
L3-4 & 22 \\
L4-5 & 21 \\
L5-S1 & \\
annulus defect & $4.9 \pm 0.70$ \\
Height, (mm) & $7.8 \pm 1.5$ \\
Width (mm) & $38.6 \pm 10.9$ \\
Area (mm ${ }^{2}$ ) & $1.6 \pm 1.1$ \\
Volume of nucleus removed, $\mathrm{mL}$ & $2.4 \pm 1.4$ \\
Length of hospitalization, days & \\
\hline
\end{tabular}

Pre- and post-operative VAS scores for back pain and ipsilateral leg pain for these 40 patients are depicted in Figure 5 (back pain) and Fig. 6 (leg pain). Significant reductions in VAS scores were noted following surgery throughout the 12 months follow-up period $(\mathrm{p}<0.0001$, Wilcoxon Rank-Sum test). ODI scores followed a similar course (Fig. 7) and were significantly lower postoperatively compared to baseline $(\mathrm{p}<0.0001$, Wilcoxon Rank-Sum test).

\section{Radiographic results}

As depicted in the patient flowchart in Fig. 4, radiographic data necessary to evaluate disc height were available for 30 of

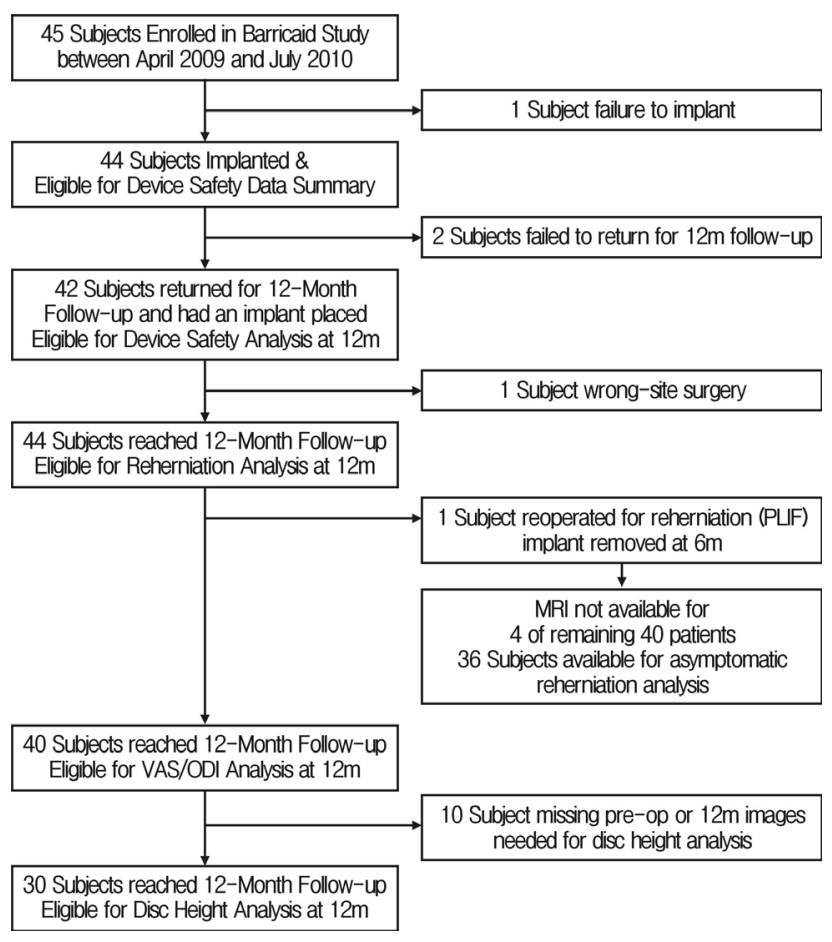

Fig. 4. Patient accounting flow-chart.

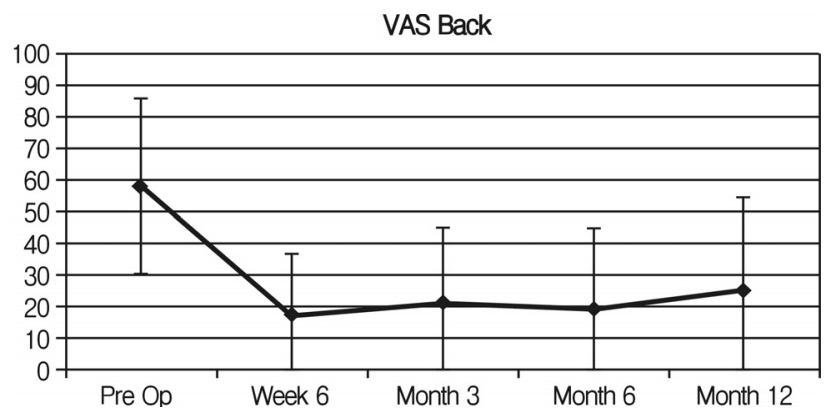

Fig. 5. Mean Visual Analog Scale (VAS) back pain scores. Error bars represent standard deviation. All post-operative time points are significantly improved compared to pre-operative. 


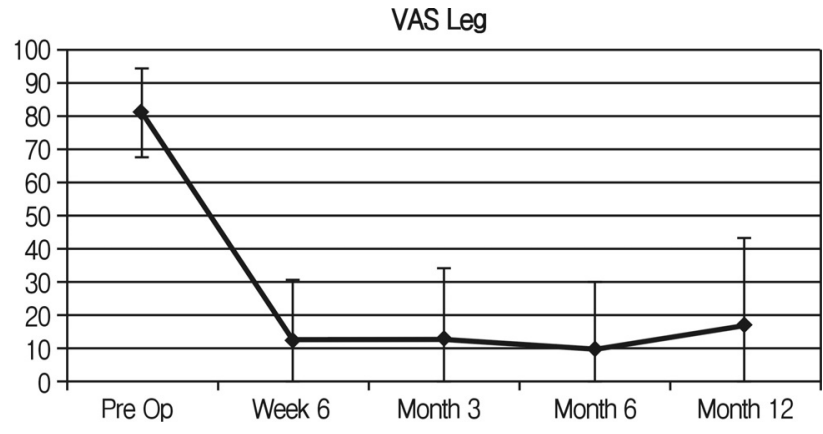

Fig. 6. Mean Visual Analog Scale (VAS) leg pain scores. Error bars represent standard deviation. All post-operative time points are significantly improved compared to pre-operative.

ODI

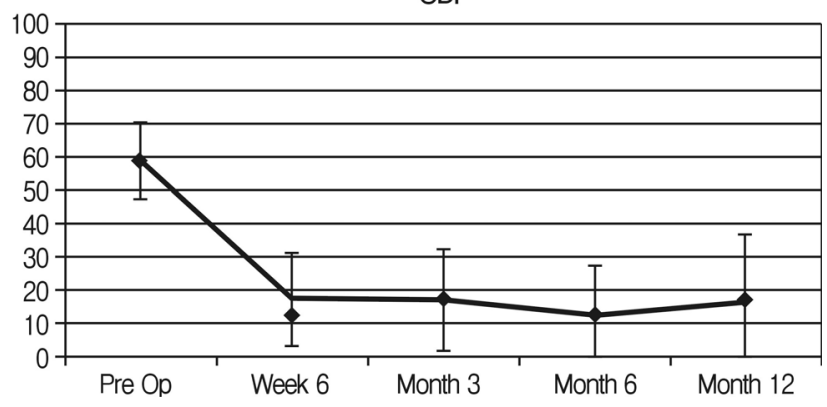

Fig. 7. Mean Oswestry Disability Index (ODI) patient function scores. Error bars represent standard deviation. All post-operative time points are significantly improved compared to pre-operative.

\section{Disc Height Maintenance}

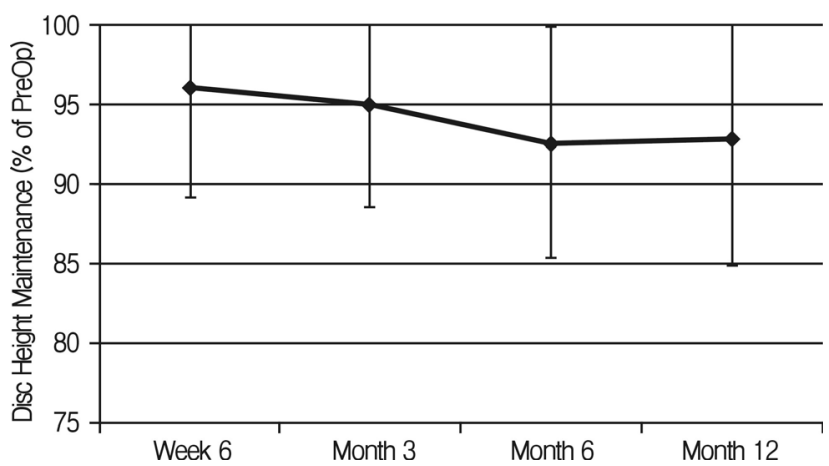

Fig. 8. Mean disc height maintenance. Error bars represent standard deviation.

these 40 patients. There was a small but significant decrease of the mean disc height to $92.8 \%$ of baseline (S.D. 7.9\%) (Fig. 8 ) at 12 months $(p<0.01$, one-sided Student t-test). No statistically significant differences were found between mean preoperative and 12-month evaluations for disc angle, spondylolisthesis, rotation and translation. Furthermore, no evidence was found of heterotopic ossification or spontaneous fusion.
Table 3. Degree of facet joint arthopathy preoperative and at 12 months according to Pathria classification ${ }^{20)}$

\begin{tabular}{llcc}
\hline \hline & \multicolumn{2}{c}{ No. of patients } \\
\cline { 2 - 3 } & Preop & 12 months \\
\hline 0 Normal & 20 & 17 \\
1 Mild - Joint space narrowing & 15 & 16 \\
2 Moderate - JJoint space narrowing + & 2 & 4 \\
& sclerosis/hypertrophy & \\
3 & Severe - Jnarrowing with sclerosis/ \\
hypertrophy and osteophytes & 2 & 1 \\
$\quad$ Total & 39 & 38 \\
\hline
\end{tabular}

Fisher's Exact: $p=0.781$ comparing grade distribution preop vs. $12 \mathrm{~m}$

Table 4. Degree of disc degeneration preoperative and at 12 months according to Pfirrmann classification ${ }^{22}$

\begin{tabular}{|c|c|c|c|}
\hline & \multicolumn{2}{|c|}{ No. of patients } \\
\hline & & \multicolumn{2}{|c|}{ Preop 12 months } \\
\hline Grade I & Bright white disc, homogenous structure & 1 & 0 \\
\hline Grade II & White disc, inhomogenous structure & 1 & 1 \\
\hline Grade III & Gray disc, inhomogenous structure & 27 & 21 \\
\hline Grade IV & $\begin{array}{l}\text { Gray to black (dark gray) disc, } \\
\text { inhomogenous structure }\end{array}$ & 13 & 14 \\
\hline Grade V & Black disc, inhomogenous structure, & 0 & 0 \\
\hline
\end{tabular}

Fisher's Exact: $p=0.857$ comparing grade distribution preop vs. $12 \mathrm{~m}$

In all cases, the device was intact, with no signs of subsidence or migration. The degree of facet joint arthopathy and disc degeneration preoperative and at 12 months are presented in Tables 3 and 4. The differences noted were not statistically significant (Fisher's Exact: $\mathrm{p}=0.781$ for facet joint arthropathy and $p=0.857$ for disc degeneration)

\section{Reherniation and other adverse events}

As depicted in the patient flowchart in Fig. 4, one (2.4\%) of the 41 subjects evaluable for reherniation at twelve months had a symptomatic reherniation and required a subsequent reo peration. The subject was a 40 year-old male who was a current smoker with a body mass index (BMI) of 22.1. Primary surgery was an L4-5 discectomy with the annulus closure device implantation performed to treat a defect with an area of $32 \mathrm{~mm}^{2}$ ( $4 \mathrm{~mm}$ tall by $8 \mathrm{~mm}$ wide) following removal of $1.3 \mathrm{~mL}$ of nucleus. Four months after surgery, the patient reported an increase in back and leg pain and a recurrent herniation was confirmed on MR. Clinical assessment at 6 months showed an 
increase in low back pain VAS (from 14/100 to 59/100); no device subsidence, migration, or spontaneous fusion was observed radiographically. The annulus closure device was remo ved and a posterior lumbar interbody fusion was performed. The operating surgeon and the DSMB independently concluded that the annulus closure device was implanted $3 \mathrm{~mm}$ too deep into the disc space, which exceeded the range in the described surgical technique, permitting the development of a nuclear extrusion around the device. This patient continues to have back pain at the 12 month follow-up, with a VAS back of 87/100, ODI 46/100, and VAS leg of 16/100.

Of the remaining 40 subjects evaluable for reherniation at 12 months, MRIs were available for 36. Review of these images revealed that one subject (2.8\%) exhibited evidence of an asymptomatic nuclear extrusion in the region of the Barricaid $^{\circledR}$, maximal $6 \mathrm{~mm}$ beyond the confines of the annulus fibrosus but still in contact with the disc on imaging. At the index surgery, subject was a 43 year-old male smoker with a BMI of 25.2. An L4-5 discectomy with annulus closure device implantation was performed to treat a defect with an area of $40 \mathrm{~mm}^{2}$ (5 mm tall by $8 \mathrm{~mm}$ wide) following removal of 2.5 $\mathrm{mL}$ of nucleus. At 12 months, clinical assessment revealed improvements in this subject's leg pain VAS (from 76/100 pre-op to 2/100 at 12 months) and Oswestry (from 48/100 to 26/ 100 ) and a slight increase in low back pain VAS (from $27 / 100$ to $32 / 100)$. This subject continues to be clinically asymptomatic and is undergoing routine monitoring.

No other reherniations were reported. Two other reoperations were performed, one for a contralateral herniation three weeks following the index surgery, and one for excessive scar tissue five months following the index surgery.

According to the review by the DSMB, no device-related adverse events have occurred in the study population. Of the 17 subjects who have reached the two-year time point, all had 24-month follow-up safety data available. None of these had evidence of recurrent disc herniation or device migration, subsidence, or fracture/disassembly. No dural tears were reported in this study

\section{DISCUSSION}

Placement of an annulus closure device following limited discectomy yielded short-term results in terms of reduced leg and back pain that were as good as those of limited discectomy alone ${ }^{3,7}$. No device-related adverse events have occurred throughout the course of study. In addition, 12-month and initial 24-month radiographic analysis revealed intact devices, with no evidence of subsidence or migration. These results support a favorable initial safety profile for this annulus closure device within 12 months following surgery.

Although a long-term study is needed to fully assess the ability of this annulus closure device to prevent recurrent reherniations, the low rate of reherniation in this series suggests success in preventing early reherniations. Only one patient had a symptomatic reherniation (2.4\%) within the first 12 months following surgery. The surgeon later determined that device malpositioning at the time of implantation in this case likely influenced the outcome, and the manufacturer has addressed this issue by developing a second generation delivery tool that limits implantation depth. A second reherniation that until now remains asymptomatic was identified on a prospective review of annual MRs by an independent radiology lab. An asymptomatic recurrence in patients treated with a limited discectomy is not unusual ${ }^{4}$. Recurrent disc prolapses without clinical symptoms may be present in as many as $13 \%$ of discectomy patients after two years following surgery ${ }^{15}$. The one asymptomatic reherniation we found in this study (2.8\%) represents a reduced rate compared to discectomy patients in whom annulus closure was not performed. In one patient, a contralateral herniation occurred at the same level within 3 weeks after the index surgery. Contralateral herniations have been reported in $4 \%$ within one year following primary discectomy ${ }^{19}$. Yet, the possibility that implantation of the Barricaid resulted in a pressure increase in the disc that contributed to rupture of the annulus at the contralateral side cannot be excluded. This question may be answered in a randomized trial.

A review of the literature demonstrates that the reported incidence of symptomatic and/or reoperated recurrent herniation within 12 months following lumbar discectomy performed by a variety of techniques ranges from $0-18.1 \% 0^{2,3,11,12,17,19,24,25)}$. Not all patients have an equivalent risk of reherniation. annulus defect size observed at the time of surgery has been implicated as a major risk factor for recurrence ${ }^{17)}$. Carragee et al. ${ }^{5)}$ reported a reherniation rate for discs with "massive" defects (>6 $\mathrm{mm}$ in width) that was almost six times greater when compared to the remaining patient cohort with smaller defects ( $27.3 \%$ vs. $4.8 \%)$, identifying this as "an independent predictor of reherniation". The vast majority (33 of 41) of annulus defects in the present study matched Carragee's definition of "massive", and were therefore at a significantly higher risk of reherniation than those with smaller defects.

The aggressive removal of nucleus from within the disc space has been a surgical technique to lower the reherniation rate, though this can lead to worse clinical outcomes ${ }^{17}$. One metaanalysis concluded that the higher reherniation rate associated with more limited discectomy $(8.7 \%$ vs. $3.3 \%$ for aggressive nuclectomy) is offset by the decreased incidence of recurrent back and leg pain $(11 . \% \text { vs. } 28 \%)^{26}$. In our study, use of an annulus closure device in patients treated with limited discec- 
tomy appears to have resulted in reherniation rates on par with more aggressive nucleus removal, but with the clinical benefits associated with retention of more of the native nucleus. These results were achieved despite a majority of patients having large annulus defects with a significantly increased risk of reherniation. It remains to be seen whether this combination of positive outcomes continues in the long-term.

The device also appears effective in maintaining disc height. The majority of discectomy patients experience greater than a $25 \%$ loss of preoperative disc height after surgery ${ }^{4,16,18,27)}$, which has been associated with poor clinical outcomes, particularly low back pain ${ }^{4,27}$. In our study, patients maintained an average of $92.8 \%$ of their pre-operative disc height at the 12 month follow-up with no significant difference between preoperative and 12-month values across the population. Volume of nucleus removed at the time of primary discectomy has been identified as a particularly significant factor associated with disc height loss following surgery ${ }^{17}$. The mean volume of nucleus removed in our study was indeed 20\% less than the volume reported by $\mathrm{McGirt}$ et $\mathrm{al}^{17)}$. In conclusion, the use of an annulus closure device may provide a reduction in reherniation rate for lumbar discectomy patients with large annulus defects who are at the greatest risk of recurrence. Using such a device should provide the surgeon increased confidence in reducing the volume of nucleus removed, which, in turn, may preserve disc height and biomechanics, reducing degeneration and associated poor clinical outcomes in the long-term.

Possible risks and complications associated with the device should also be considered. Implantation requires sufficient space to place the device into the disc defect without undue retraction of the nerve root while protecting the dura. This may be a problem with central disc herniations unless the dura and nerve root can be mobilized sufficiently. In the present series the device was used in central disc defects in 3 cases without complications. No dural tears or nerve root damage occurred in any patient. As with any implant, there is a risk of device migration, subsidence, loosening or fracture, and allergic reaction by the patient. None of these complications were encountered in this series so far. Scar formation may be another concern related to the introduction of a foreign body and associated surgical manipulations. In the present series, one patient had to be reoperated for excessive scar tissue. Scar tissue formation, however, is a relatively common finding after lumbar discectomy, the procedure for which the studied device is an adjunct. Kotilainen et al. reported that 4 of 237 (1.7\%) discectomy patients required reoperation due to excessive scar tissue formation, and Daneyemez et al. reported that 26 of 1072 (2.4\%) of discectomy patients required reoperation for epidural fibrosis, which is within the same rangeas in our study $(1 / 45=2.2 \%)^{9,14)}$. A randomized controlled study will provide more information on the relative contribution of the annulus closure device to postoperative scarring.

Main study strengths include the prospective, multi-center design, use of a standard protocol with strict inclusion and exclusion criteria for patient selection, use of a standardized surgical technique and assessment tools, and independent evaluation of imaging and adverse events. Methodological weaknesses include lack of randomization, small sample size, and only preliminary follow-up. A prospective, randomized, multicenter study evaluating limited discectomy with and without the use of the annulus closure device is currently underway, and will provide a higher level of evidence regarding the device's safety and efficacy.

\section{REFERENCES}

1. Adams MA, McNally DS, Dolan P: 'Stress' distributions inside intervertebral discs. The effects of age and degeneration. J Bone Joint Surg Br 78:965-972, 1996

2. Ambrossi GL, McGirt MJ, Sciubba DM, Witham TF, Wolinsky JP, Gokaslan ZL, et al: Recurrent lumbar disc herniation after single-level lumbar discectomy: incidence and health care cost analysis. Neurosurgery 65:574-578, 2009

3. Arts MP, Brand R, van den Akker ME, Koes BW, Bartels RH, Peul WC: Tubular diskectomy vs conventional microdiskectomy for sciatica: a randomized controlled trial. JAMA 302:149-158, 2009

4. Barth M, Diepers M, Weiss C, Thome C: Two-year outcome after lumbar microdiscectomy versus microscopic sequestrectomy: part 2: radiographic evaluation and correlation with clinical outcome. Spine 33:273-279, 2008

5. Carragee EJ, Han MY, Suen PW, Kim D: Clinical outcomes after lumbar discectomy for sciatica: the effects of fragment type and annulus competence. J Bone Joint Surg Am 85-A:102-108, 2003

6. Carragee EJ, Han MY, Yang B, Kim DH, Kraemer H, Billys J: Activity restrictions after posterior lumbar discectomy. A prospective study of outcomes in 152 cases with no postoperative restrictions. Spine 24:2346-2351, 1999

7. Carragee EJ, Spinnickie AO, Alamin TF, Paragioudakis S: A prospective controlled study of limited versus subtotal posterior discectomy: short-term outcomes in patients with herniated lumbar intervertebral discs and large posterior annulus defect. Spine 31: 653-657, 2006

8. Caspar W, Campbell B, Barbier DD, Kretschmmer R, Gotfried $\mathrm{Y}$ : The Caspar microsurgical discectomy and comparison with a conventional standard lumbar disc procedure. Neurosurgery 28:78-86, 1991

9. Daneyemez M, Sali A, Kahraman S, Beduk A, Seber N: Outcome analyses in 1072 surgically treated lumbar disc herniations. Minim Invasive Neurosurg 42:63-68, 1999

10. Dunlop RB, Adams MA, Hutton WC: Disc space narrowing and the lumbar facet joints. J Bone Joint Surg Br 66:706-710, 1984

11. Garg B, Nagraja UB, Jayaswal A: Microendoscopic versus open discectomy for lumbar disc herniation: a prospective randomised study. J Orthop Surg (Hong Kong) 19:30-34, 2011 
12. Gaston P, Marshall RW: Survival analysis is a better estimate of recurrent disc herniation. J Bone Joint Surg Br 85:535-537, 2003

13. Heuer F, Ulrich S, Claes L, Wilke HJ: Biomechanical evaluation of conventional anulus fibrosus closure methods required for nucleus replacement. Laboratory investigation. J Neurosurg Spine 9:307-313, 2008

14. Kotilainen E, Valtonen S, Carlson CA: Microsurgical treatment of lumbar disc herniation: follow-up of 237 patients. Acta Neurochir (Wien) 120:143-149, 1993

15. Lebow RL, Adogwa O, Parker SL, Sharma A, Cheng J, McGirt MJ: Asymptomatic same-site recurrent disc herniation after lumbar discectomy: results of a prospective longitudinal study with 2-year serial imaging. Spine 36:2147-2151, 2011

16. Loupasis GA, Stamos K, Katonis PG, Sapkas G, Korres DS, Hartofilakidis G: Seven- to 20-year outcome of lumbar discectomy. Spine 24:2313-2317, 1999

17. McGirt MJ, Ambrossi GL, Datoo G, Sciubba DM, Witham TF, Wolinsky JP, et al: Recurrent disc herniation and long-term back pain after primary lumbar discectomy: review of outcomes reported for limited versus aggressive disc removal. Neurosurgery 64: 338-344, 2009

18. McGirt MJ, Eustacchio S, Varga P, Vilendecic M, Trummer M, Gorensek M, et al: A prospective cohort study of close interval computed tomography and magnetic resonance imaging after primary lumbar discectomy: factors associated with recurrent disc herniation and disc height loss. Spine 34:2044-2051, 2009

19. Nystrom B: Experience of microsurgical compared with conventional technique in lumbar disc operations. Acta Neurol Scand
76:129-141, 1987

20. Pathria M, Sartoris DJ, Resnick D: Osteoarthritis of the facet joints: accuracy of oblique radiographic assessment. Radiology 164:227-230, 1987

21. Peul WC, van den Hout WB, Brand R, Thomeer RT, Koes BW: Prolonged conservative care versus early surgery in patients with sciatica caused by lumbar disc herniation: two year results of a randomised controlled trial. BMJ 336:1355-1358, 2008

22. Pfirrmann CW, Metzdorf A, Zanetti M, Hodler J, Boos N: Magnetic resonance classification of lumbar intervertebral disc degeneration. Spine 26:1873-1878, 2001

23. Reitman CA, Mauro KM, Nguyen L, Ziegler JM, Hipp JA: Intervertebral motion between flexion and extension in asymptomatic individuals. Spine 29:2832-2843, 2004

24. Rogers LA: Experience with limited versus extensive disc removal in patients undergoing microsurgical operations for ruptured lumbar discs. Neurosurgery 22:82-85, 1988

25. Thome C, Barth M, Scharf J, Schmiedek P: Outcome after lumbar sequestrectomy compared with microdiscectomy: a prospective randomized study. J Neurosurg Spine 2:271-278, 2005

26. Watters WC, III, McGirt MJ: An evidence-based review of the literature on the consequences of conservative versus aggressive discectomy for the treatment of primary disc herniation with radiculopathy. Spine J 9:240-257, 2009

27. Yorimitsu E, Chiba K, Toyama Y, Hirabayashi K: Long-term outcomes of standard discectomy for lumbar disc herniation: a follow-up study of more than 10 years. Spine 26:652-657, 2001 